
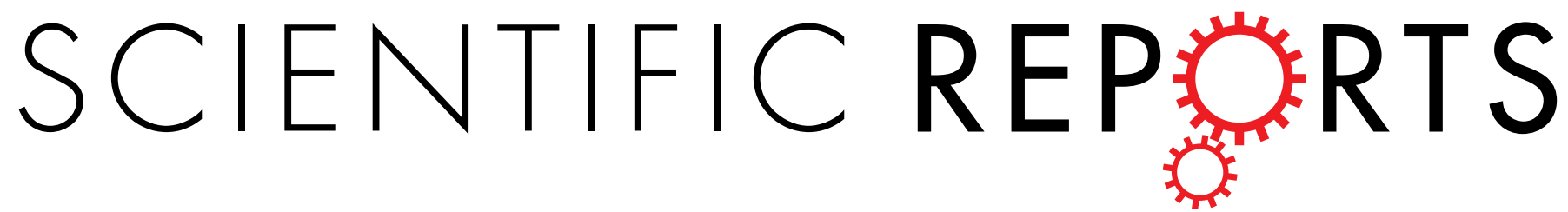

\title{
OPEN Associations of Plasma FGF2 Levels and Polymorphisms in the FGF2 Gene with Obesity Phenotypes in Han Chinese Population
}

Received: 14 July 2015

Accepted: 18 December 2015

Published: 16 February 2016

\author{
Ruo-Han Hao ${ }^{1}$, Yan Guo ${ }^{1}$, Shan-Shan Dong ${ }^{1}$, Gai-Zhi Weng ${ }^{2}$, Han Yan ${ }^{1}$, Dong-Li Zhu ${ }^{1}$, \\ Xiao-Feng Chen ${ }^{1}$, Jia-Bin Chen ${ }^{1}$ \& Tie-Lin Yang ${ }^{1}$
}

Obesity is highly heritable, but the specific genes influencing obesity related traits are largely unknown. Fibroblast growth factor 2 (FGF2) could influence adipocyte differentiation. However, the association of FGF2 polymorphisms and obesity remains unclear. This study aimed to investigate the associations of both the plasma FGF2 levels and SNPs in FGF2 gene with obesity phenotypes in Han Chinese populations. Plasma FGF2 levels were measured and subjected to association analyses in 62 subjects. Eleven SNPs in FGF2 were genotyped and tested for associations in a discovery sample of 1,300 subjects. SNPs significantly associated with obesity were subjected to replication in another independent sample of 1,035 subjects. We found that plasma FGF2 levels were positively correlated with fat mass $(P=0.010)$. Association analyses in the discovery sample identified three SNPs (rs1449683, rs167428, rs308442) significantly associated with fat mass after multiple testing adjustments $(P<0.0045)$. Subsequent replication study successfully validated one SNP (rs167428) associated with fat mass $\left(P_{\text {combine }}=3.46 \times 10^{-5}\right)$. eQTL analyses revealed that SNPs associated with obesity also affected $F G F 2$ expression. Our findings suggested that high plasma FGF2 level correlated with increased risk of obesity, and FGF2 gene polymorphisms could affect individual variances of obesity in Han Chinese population.

Obesity is a major worldwide health problem associated with increased risk of type 2 diabetes, heart diseases, and several forms of cancer ${ }^{1}$. The prevalence of obesity continues to increase at an alarming rate. In China, $32.1 \%$ of Chinese are overweight, and $9.9 \%$ are obese according to the National Physique Monitoring Bulletin in 2010 (http://www.gov.cn/test/2012-04/19/content_2117320.htm). In the Chinese population, individuals with a $\mathrm{BMI} \geq 23 \mathrm{~kg} / \mathrm{m}^{2}$ are considered as overweight, and those with a BMI $\geq 27.5 \mathrm{~kg} / \mathrm{m}^{2}$ are obese ${ }^{2}$.

Obesity is a typical complex disease of strong genetic determination, with heritability estimated ranging from 40 to $70 \% \%^{3,4}$. In the past years, genome-wide association studies (GWASs) have successfully identified a number of genetic determinants for obesity, such as $F T O^{5}, I N S I G 2^{6}$, and $M C 4 R^{6}$, etc. However, the identified loci together only account for a small proportion of the genetic predisposition $(<10 \%)$ to obesity, suggesting that many more associated variants remain to be discovered.

FGF2 gene is located in chromosome 4q26, and encodes a member of the fibroblast growth factors (FGFs). FGFs are involved in numerous biological processes, including adipogenesis ${ }^{7}$. FGF2, one of the most often investigated cytokines in FGFs, can be synthesized and secreted by human adipocytes ${ }^{8}$. Previous functional studies revealed a close relationship between FGF2 and adipocytes. For example, Kawaguchi et al. ${ }^{9}$ found that FGF2 can induce the de novo adipogenesis with reconstituted basement membrane supplied, suggesting that FGF2 can influence the distribution of adipose tissue in the body, which in turn affect the risk for metabolic complications. Kakudo et al. ${ }^{10}$ discovered that FGF2 significantly enhanced the adipogenic differentiation of adipose-derived stem cells into adipocytes. Given that the major definition of obesity is the excessive proliferation of adipocytes, it reminds us that FGF2 might be related with obesity and FGF2 gene could be a new candidate gene for obesity.

${ }^{1}$ Key Laboratory of Biomedical Information Engineering of Ministry of Education, School of Life Science and Technology, Xi'an Jiaotong University, Xi'an 710049, P. R. China. 'Laboratory of Xi'an Jiaotong University Hospital, Xi'an 710049, P. R. China. Correspondence and requests for materials should be addressed to T.-L.Y. (email: yangtielin@mail.xjtu.edu.cn) 


\begin{tabular}{|l|c|c|c|}
\hline Trait & Plasma Sample & Discovery Sample & Replication Sample \\
\hline Number & 62 & 1,300 & 1,035 \\
\hline Male/Female & $18 / 44$ & $600 / 700$ & $345 / 690$ \\
\hline Age (years) & $63.08(6.84)$ & $33.42(11.32)$ & $50.36(17.75)$ \\
\hline Weight $(\mathrm{kg})$ & $62.40(9.98)$ & $59.63(10.41)$ & $61.72(10.32)$ \\
\hline Height $(\mathrm{cm})$ & $159.94(7.77)$ & $163.94(8.11)$ & $161.85(7.99)$ \\
\hline BMI $\left(\mathrm{kg} / \mathrm{m}^{2}\right)$ & $24.31(2.81)$ & $22.11(2.98)$ & $23.53(3.28)$ \\
\hline Fat Mass $(\mathrm{kg})$ & $19.53(7.04)$ & $13.99(5.38)$ & $16.21(7.10)$ \\
\hline Hip circumference $(\mathrm{m})$ & - & $0.92(0.06)$ & $0.96(0.07)$ \\
\hline Waist circumference $(\mathrm{m})$ & - & $0.75(0.09)$ & $0.79(0.11)$ \\
\hline Waist-hip ratio & - & $0.80(0.07)$ & $0.82(0.08)$ \\
\hline Plasma FGF2 $(\mathrm{ng} / \mathrm{L})$ & $23.27(23.39)$ & - & - \\
\hline
\end{tabular}

Table 1. Basic characteristics of the study samples. Note: Normally distributed data are shown as mean (standard deviation, SD).

FGF2 concentration/(ng/L)

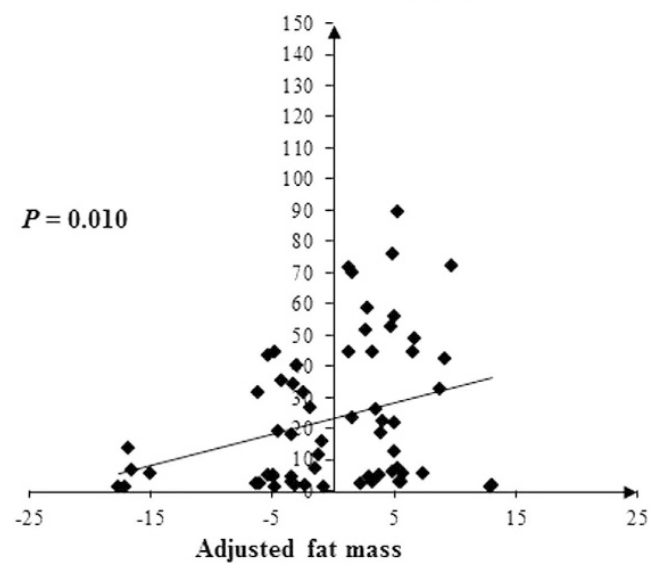

a

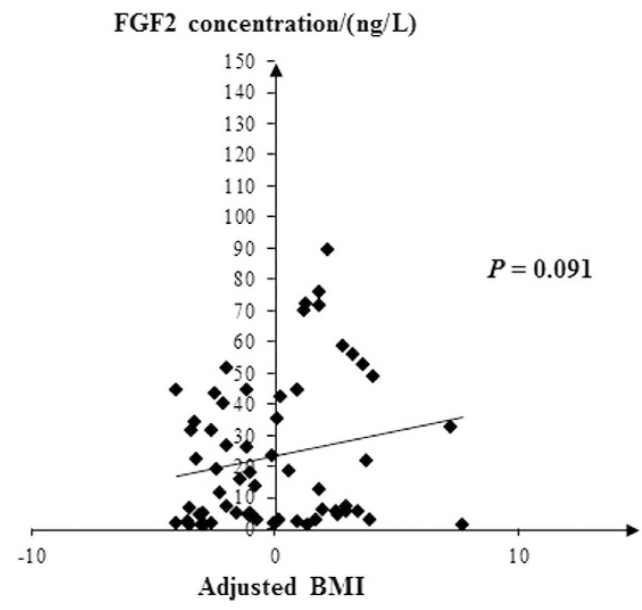

b

Figure 1. Correlation plots of plasma levels of FGF2 after adjusting for sex and age with (a) fat mass and (b) BMI.

Till now, only one study indicated a negative correlation between serum FGF2 levels and BMI in 30 Japanese overweight men ${ }^{11}$. Recently, Shungin et al. has reported a SNP (rs303084) near FGF2 as a new loci associated with waist-hip ratio adjusting for BMI (WHRadjBMI) ${ }^{12}$, as the only genetic association evidence to reveal the relationship of FGF2 with obesity. Considering the limited findings, the level of FGF2 and genetic basis in obesity still remain poorly understood. Therefore, the aims of this study were as follows: 1) to evaluate the associations of plasma FGF2 levels with obese status and 2) to investigate the relationship of SNPs in FGF2 with obesity related traits in Han Chinese populations.

\section{Results}

Correlation analyses of plasma FGF2 levels with obese status. We performed correlation analyses of plasma FGF2 levels with obesity phenotypes, including BMI and body fat mass, in 62 unrelated Han Chinese subjects. The basic characteristics of this sample are summarized in Table 1. For the plasma sample, the profile of FGF2 level was $23.27 \pm 23.39 \mathrm{ng} / \mathrm{L}$, and the correlations between FGF2 level and obesity phenotypes are particularly shown in scatter plots in Fig. 1. Significant positive correlation was observed between FGF2 levels and fat mass $(\beta=0.092( \pm 0.026), P=0.015)$, which remained significant even after adjusting for age and $\operatorname{sex}(\beta=0.096$ $( \pm 0.025), P=0.010)$. However, we did not observe significant association of FGF2 levels with adjusted BMI $(\beta=0.025$ ( \pm 0.015$), P=0.091)$, even though the association was in the same direction as that with fat mass.

Association of SNPs in FGF2 with obesity related traits in the discovery sample. We selected 11 SNPs in FGF2 to test for associations with obesity-related traits (BMI, fat mass and WHRadjBMI) in the discovery sample of 1,300 Han Chinese subjects. The basic characteristics of this sample are shown in Table 1. SNPs and the major association results are summarized in Table 2. For BMI, only 1 SNP showed nominal significant association (rs167428: $P=0.019)$. For fat mass, 8 SNPs presented nominal significant associations $(P<0.05)$. For WHRadjBMI, SNP rs308387 reached nominal significance $(P=0.041)$. After Bonferroni correction, 3 SNPs remained significant for association with fat mass, including rs $1449683\left(P=1.11 \times 10^{-4}\right)$, rs167428 $\left(P=1.67 \times 10^{-4}\right)$, and $\mathrm{rs} 308442\left(P=5.20 \times 10^{-4}\right)$. Each minor allele of these 3 SNPs was associated 


\begin{tabular}{|c|c|c|c|c|c|c|c|c|c|c|c|c|c|}
\hline \multirow[b]{2}{*}{ No. } & \multirow[b]{2}{*}{ SNP } & \multirow{2}{*}{$\begin{array}{l}\text { Physical } \\
\text { Position }\end{array}$} & \multirow{2}{*}{$\begin{array}{c}\text { Genic } \\
\text { Position }\end{array}$} & \multirow[b]{2}{*}{$\mathbf{A} 1 / \mathbf{A} 2^{\mathbf{a}}$} & \multirow[b]{2}{*}{ MAF } & \multicolumn{2}{|c|}{ BMI } & \multicolumn{2}{|c|}{ Fat Mass } & \multicolumn{2}{|c|}{ WHRadjBMI } & \multirow[b]{2}{*}{$\boldsymbol{\beta}_{\text {еQTL }}{ }^{\mathrm{b}}$} & \multirow[b]{2}{*}{$P_{\mathrm{eQTL}^{\mathrm{b}}}^{\mathrm{b}}$} \\
\hline & & & & & & $\beta$ & $P$ & $\beta$ & $P$ & $\beta$ & $P$ & & \\
\hline 1 & rs1449683 & 123748086 & Exon1 & $\mathrm{T} / \mathrm{C}$ & 0.165 & -0.255 & 0.061 & -0.877 & $1.11 \times 10^{-4}$ & $-3.03 \times 10^{-3}$ & 0.261 & -0.059 & $4.86 \times 10^{-3}$ \\
\hline 2 & rs308403 & 123757748 & Intron1 & $\mathrm{C} / \mathrm{T}$ & 0.320 & 0.170 & 0.140 & 0.251 & 0.193 & $1.54 \times 10^{-3}$ & 0.501 & - & - \\
\hline 3 & rs308418 & 123768263 & Intron1 & $\mathrm{A} / \mathrm{G}$ & 0.157 & 0.139 & 0.340 & 0.124 & 0.610 & $5.38 \times 10^{-4}$ & 0.852 & -0.030 & 0.288 \\
\hline 4 & rs167428 & 123773439 & Intron1 & $\mathrm{C} / \mathrm{T}$ & 0.120 & -0.367 & 0.019 & -0.986 & $1.67 \times 10^{-4}$ & $-3.10 \times 10^{-3}$ & 0.321 & -0.059 & $2.05 \times 10^{-5}$ \\
\hline 5 & rs308442 & 123774913 & Intron1 & $\mathrm{A} / \mathrm{T}$ & 0.305 & -0.202 & 0.107 & -0.724 & $5.20 \times 10^{-4}$ & $-1.21 \times 10^{-3}$ & 0.625 & -0.065 & $1.40 \times 10^{-6}$ \\
\hline 6 & rs3789138 & 123784721 & Intron1 & G/A & 0.500 & -0.127 & 0.212 & -0.377 & 0.027 & $1.66 \times 10^{-3}$ & 0.412 & 0.070 & $5.09 \times 10^{-6}$ \\
\hline 7 & rs308387 & 123786832 & Intron1 & $\mathrm{A} / \mathrm{G}$ & 0.043 & -0.115 & 0.651 & -0.299 & 0.482 & $-1.03 \times 10^{-2}$ & 0.041 & - & - \\
\hline 8 & rs12506710 & 123794127 & Intron1 & $\mathrm{C} / \mathrm{T}$ & 0.445 & 0.157 & 0.122 & 0.419 & 0.013 & $-9.34 \times 10^{-5}$ & 0.963 & - & - \\
\hline 9 & rs10011927 & 123798428 & Intron2 & $\mathrm{C} / \mathrm{T}$ & 0.449 & 0.155 & 0.127 & 0.427 & 0.011 & $5.77 \times 10^{-5}$ & 0.977 & - & - \\
\hline 10 & rs1048201 & 123814308 & $3^{\prime}$ UTR & $\mathrm{T} / \mathrm{C}$ & 0.447 & 0.173 & 0.089 & 0.450 & $7.92 \times 10^{-3}$ & $-2.06 \times 10^{-4}$ & 0.919 & 0.046 & 0.010 \\
\hline 11 & rs1476217 & 123818511 & $3^{\prime}$ UTR & $\mathrm{C} / \mathrm{A}$ & 0.452 & 0.157 & 0.135 & 0.432 & 0.013 & $3.44 \times 10^{-4}$ & 0.869 & -0.016 & 0.331 \\
\hline
\end{tabular}

Table 2. Association results for 11 SNPs in FGF2 with BMI, fat mass and WHRadjBMI in the Chinese discovery sample. Note: MAF, minor allele frequency. $\beta$ is calculated with respect to the minor allele. Significant $P$ values after multiple testing adjustment $(P<0.0045)$ are shown in bold. ${ }^{\text {a }} \mathrm{A} 1$ represents the minor allele. ${ }^{\mathrm{b}} \beta_{\mathrm{eQTL}}$ and $P_{\mathrm{eQTL}}$ are shown for 210 HapMap unrelated individuals from 4 populations. $\beta_{\mathrm{eQTL}}$ is calculated with respect to the minor allele.

with reduced fat mass values with the effect size $(\beta)$ of -0.877 (rs1449683-T), -0.986 (rs167428-C), and -0.724 (rs308442-A), respectively. As for their genetic positions, rs1449683 is located at the exon 1 of FGF2, while the other two (rs167428 and rs308442) are close to each other and located at the intron 1. We analyzed the linkage disequilibrium (LD) between rs167428 and rs308442, and found that these two SNPs were in modest LD with each other (pair wise $r^{2}=0.31, D^{\prime}=1.0$ ). We then performed conditional analysis on rs 167428 using rs 308442 as a covariate to see the independent association effect. The $P$ value changed from 0.019 to 0.076 for BMI, and from $1.67 \times 10^{-4}$ to 0.025 for fat mass, respectively. Such results presented significant drop of association signals compared with the regular association analysis, suggesting that the association signals between these two SNPs were highly correlated.

We further characterized the LD blocks and haplotypes for SNPs in FGF2. As shown in Fig. 2, three blocks in high LD and 10 haplotypes were identified. Nine of these haplotypes were with frequencies more than 0.05 (Fig. 2b). Haplotype association results are listed in Table 3. After multiple testing adjustment, three haplotypes were significantly associated with fat mass, including haplotype "TTG" in block $1\left(P=8.71 \times 10^{-5}, \beta=-0.904\right)$, "TA" $\left(P=1.39 \times 10^{-4}, \beta=-0.999\right)$ and "CT" $\left(P=5.20 \times 10^{-4}, \beta=0.724\right)$ in block 2 . Given the 3 significant SNPs included in block 1 (rs1449683) and block 2 (rs167428 and rs308442) separately, haplotype-based association results corroborated the single SNP results.

Follow-up replication analysis. To further validate the SNP association results, we selected the top 4 significant SNPs (including the three significant SNPs (rs1449683, rs167428, and rs308442) after Bonferroni correction and one SNP rs1048201 located at 3'UTR with nearly significant signal) recognized from the discovery study for replication analyses in another independent Han Chinese sample. However, since the SNP rs 1449683 failed genotyping, we had only three SNPs for subsequent analysis. The association results are summarized in Table 4 . SNP rs167428 was successfully replicated by showing significant association signal with fat mass $(P=0.044)$ and borderline significant association signal with BMI $(P=0.053)$. The effect was in the same direction as that in the discovery sample. After meta-analysis, the combined $P$ value of rs 167428 related to fat mass achieved a much more significant level of $3.46 \times 10^{-5}$. We further performed heterogeneity test between our two samples, and no heterogeneity for rs 167428 was observed, neither for BMI $(P=0.902)$ nor for Fat Mass $(P=0.262)$.

We also checked the association results for the above SNPs in the published data on GWASs of obesity from the GIANT ${ }^{12}$ consortium and related traits like type 2 diabetes (T2D) from the DIAGRAM ${ }^{13}$ consortium. In the GIANT Consortium dataset consisted of 210,088 European individuals, SNP rs308442 was significantly associated with WHRadjBMI $(P=0.024)$ and hip circumference adjusting for BMI (HIPadjBMI) $(P=0.025)$ in females. SNP rs1048201 was significantly associated with WHRadjBMI $(P=0.037)$ and HIPadjBMI $\left(P=3.8 \times 10^{-4}\right)$ in the total sample, and this SNP was also associated with T2D risk $(P=0.01)$ in the DIAGRAMv3 dataset comprised of 12,171 T2D cases and 56,862 controls of European descent.

eQTL analysis results. eQTL analysis was performed to investigate the relevance between the identified SNPs and FGF2 gene expression levels in 210 HapMap individuals. As shown in Table 3, for all the 210 subjects, we identified 5 SNPs (rs1449683, rs167428, rs308442, rs3789138, and rs1048201) significantly associated with FGF2 mRNA expression $\left(P_{\text {eQTL }} \leq 0.01\right)$. For rs 167428 , the effect size $(\beta)$ is -0.059 for minor allele $\mathrm{C}$, with the same direction as genetic association findings. Such observation indicated that this polymorphism could result in the decrease of both FGF2 expression level and the risk of being obese. Moreover, since all the replication tested SNPs (rs167428, rs308442 and rs1048201) passed the eQTL analysis, we further performed association analyses for these three SNPs with plasma FGF2 levels in our 62 Han Chinese subjects. However, no significant signal was observed (rs167428: $P=0.133$; rs308442: $P=0.761$; and rs 1048201: $P=0.462$ ). 


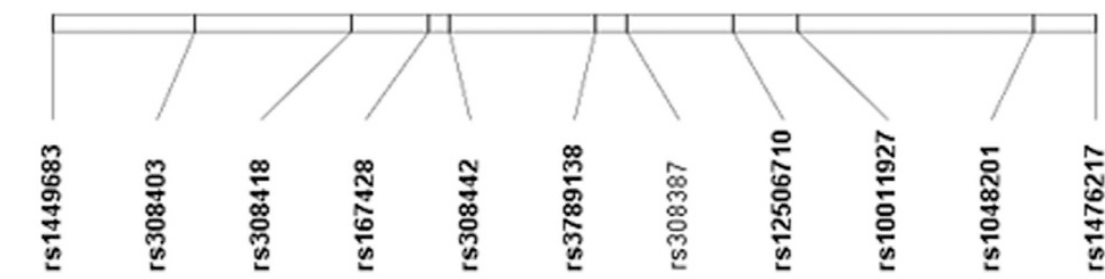

a

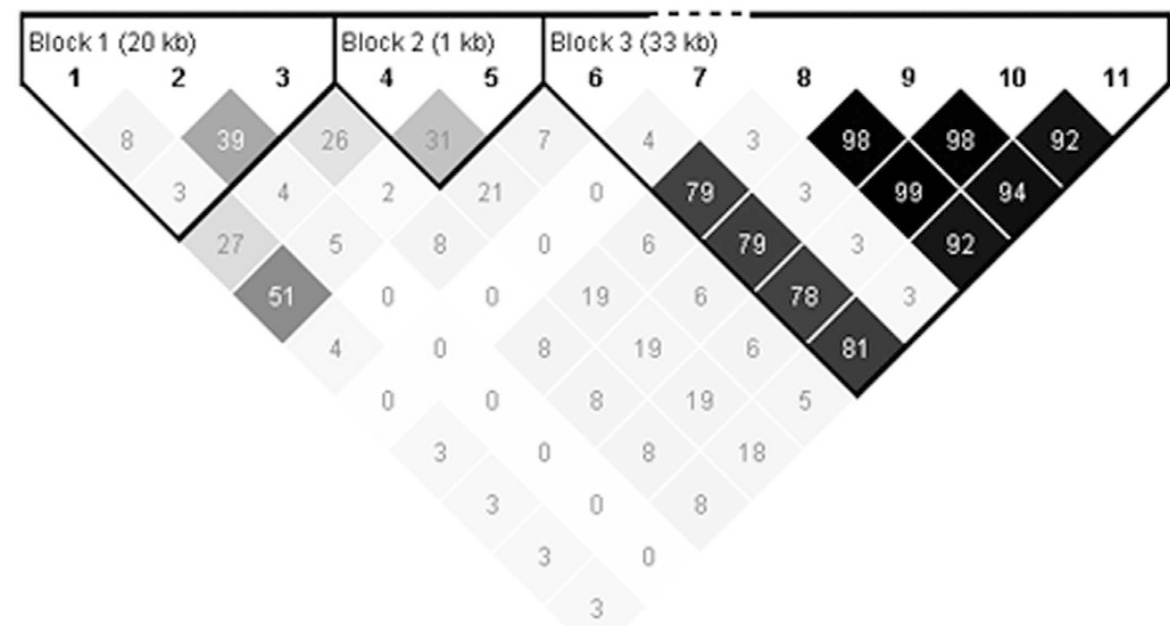

b

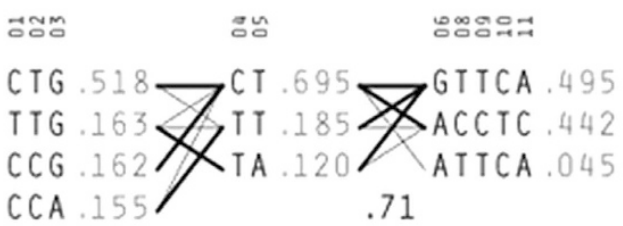

.80

Figure 2. Linkage disequilibrium (LD) blocks and haplotype frequencies for FGF2 in the discovery sample. (a) LD blocks are marked with triangles. Squares color scheme represents the value of $r^{2}$, and the black color indicates strong LD. (b) Haplotypes in the three blocks across FGF2. The haplotype frequencies are shown to the right of each haplotype. The SNP numbers correspond to those in Table 2.

\begin{tabular}{|c|c|c|c|c|c|c|c|c|c|}
\hline \multirow[b]{2}{*}{ Block } & \multirow[b]{2}{*}{ SNPs $^{\mathrm{a}}$} & \multirow[b]{2}{*}{ Haplotype $^{\mathrm{b}}$} & \multirow[b]{2}{*}{ Frequency } & \multicolumn{2}{|c|}{ BMI } & \multicolumn{2}{|c|}{ Fat Mass } & \multicolumn{2}{|c|}{ WHRadjBMI } \\
\hline & & & & $\beta$ & $P$ & $\beta$ & $P$ & $\beta$ & $P$ \\
\hline \multirow{4}{*}{ Block 1} & \multirow{4}{*}{$1-3$} & $\mathrm{CCA}$ & 0.155 & 0.144 & 0.328 & 0.132 & 0.592 & $1.03 \times 10^{-3}$ & 0.723 \\
\hline & & CCG & 0.162 & 0.132 & 0.374 & 0.273 & 0.270 & $1.62 \times 10^{-3}$ & 0.579 \\
\hline & & TTG & 0.162 & -0.268 & 0.053 & -0.904 & $8.71 \times 10^{-5}$ & $-4.36 \times 10^{-3}$ & 0.109 \\
\hline & & CTG & 0.517 & 0.018 & 0.871 & 0.348 & 0.055 & $1.20 \times 10^{-3}$ & 0.574 \\
\hline \multirow{3}{*}{ Block 2} & \multirow{3}{*}{4,5} & TA & 0.120 & -0.374 & 0.017 & -0.999 & $1.39 \times 10^{-4}$ & $-4.23 \times 10^{-3}$ & 0.172 \\
\hline & & TT & 0.185 & 0.048 & 0.745 & -0.127 & 0.605 & $1.73 \times 10^{-3}$ & 0.548 \\
\hline & & CT & 0.695 & 0.202 & 0.107 & 0.724 & $5.20 \times 10^{-4}$ & $1.41 \times 10^{-3}$ & 0.566 \\
\hline \multirow{2}{*}{ Block 3} & \multirow{2}{*}{$6,8-11$} & ACCTC & 0.440 & 0.167 & 0.103 & 0.440 & $9.82 \times 10^{-3}$ & $-1.71 \times 10^{-4}$ & 0.932 \\
\hline & & GTTCA & 0.495 & -0.121 & 0.237 & -0.376 & 0.027 & $2.09 \times 10^{-3}$ & 0.299 \\
\hline
\end{tabular}

Table 3. Haplotype identification and association analyses in the discovery sample. Significant $P$ values after multiple testing adjustment $(P<0.0056)$ are shown in bold. ${ }^{\text {TT }}$ The IDs of SNPs correspond to those in Table 2.

${ }^{\mathrm{b}}$ Haplotypes with estimated frequencies more than 0.05 are listed.

\section{Discussion}

Previous studies established FGF2 protein as a positive regulator of human preadipocytes proliferation and differentiation ${ }^{14}$. For in vivo evidence, injection of FGF2 and basement membrane in mice resulted in de novo 


\begin{tabular}{|c|c|c|c|c|c|c|c|c|c|c|c|c|c|c|c|c|c|}
\hline \multirow[b]{2}{*}{ SNP } & \multirow[b]{2}{*}{$\mathbf{A} 1 / \mathbf{A 2}^{\mathrm{a}}$} & \multirow[b]{2}{*}{ MAF } & \multicolumn{4}{|c|}{ BMI } & \multicolumn{4}{|c|}{ Fat Mass } & \multicolumn{4}{|c|}{ WHRadjBMI } & \multicolumn{2}{|c|}{ GIANT $^{\mathrm{b}}$} & \multirow[b]{2}{*}{$\begin{array}{c}\text { DIA- } \\
\text { GRAM }^{c}\end{array}$} \\
\hline & & & $\boldsymbol{\beta}$ & $P$ & $\boldsymbol{\beta}_{\text {meta }}$ & $P_{\text {meta }}$ & $\boldsymbol{\beta}$ & $P$ & $\boldsymbol{\beta}_{\text {meta }}$ & $P_{\text {meta }}$ & $\beta$ & $P$ & $\beta_{\text {meta }}$ & $P_{\text {meta }}$ & $\begin{array}{c}\text { WHRad- } \\
\text { jBMI }\end{array}$ & $\begin{array}{c}\text { HIPad- } \\
\text { jBMI }\end{array}$ & \\
\hline rs167428 & $\mathrm{C} / \mathrm{T}$ & 0.110 & -0.385 & 0.053 & -0.374 & $2.48 \times 10^{-3}$ & -0.684 & \begin{tabular}{|l|}
0.044 \\
\end{tabular} & -0.888 & $3.46 \times 10^{-5}$ & $-3.34 \times 10^{-3}$ & 0.402 & $-3.90 \times 10^{-3}$ & \begin{tabular}{|l|}
0.111 \\
\end{tabular} & 0.44 & 0.15 & 0.29 \\
\hline rs308442 & $\mathrm{A} / \mathrm{T}$ & \begin{tabular}{|l|}
0.196 \\
\end{tabular} & -0.083 & 0.595 & \begin{tabular}{|l|}
-0.156 \\
\end{tabular} & 0.111 & \begin{tabular}{|l|}
-0.099 \\
\end{tabular} & \begin{tabular}{|l|}
0.710 \\
\end{tabular} & -0.517 & $2.38 \times 10^{-3}$ & $-2.57 \times 10^{-3}$ & 0.414 & $-1.90 \times 10^{-3}$ & \begin{tabular}{|l|}
0.339 \\
\end{tabular} & 0.19 & $6.8 \times 10^{-2}$ & 0.18 \\
\hline rs1048201 & $\mathrm{T} / \mathrm{C}$ & \begin{tabular}{|l|}
0.442 \\
\end{tabular} & -0.261 & 0.040 & 0.005 & 0.951 & -0.481 & \begin{tabular}{|l|}
0.044 \\
\end{tabular} & \begin{tabular}{|l|}
0.139 \\
\end{tabular} & 0.314 & $-1.56 \times 10^{-3}$ & 0.533 & $-1.00 \times 10^{-3}$ & \begin{tabular}{|l|}
0.529 \\
\end{tabular} & 0.037 & $3.8 \times 10^{-4}$ & 0.01 \\
\hline
\end{tabular}

Table 4. Association results of 3 tested SNPs with obesity-related traits in the replication sample, GIANT and DIAGRAM Consortiums. MAF, minor allele frequency. $\beta$ is calculated with respect to the minor allele. Meta-analysis was performed with effect size estimates weighted by the inverse of the corresponding standard errors ( $\beta$ meta and Pmeta). ${ }^{a}$ A1 represents the minor allele. ${ }^{b}$ WHRadjBMI and HIPadjBMI association $P$ values are from the GIANT Consortium of 210,088 European individuals. ${ }^{c} P$ values are from the DIAGRAM Consortium of 12,171 T2D cases and 56,862 controls of European descent.

adipogenesis ${ }^{9}$. Given the biological function of FGF2 protein involved in adipocytes, it is reasonable to hypothesize that FGF2 could influence adipose-related trait.

To test the hypothesis, firstly, we performed association tests to determine whether plasma FGF2 was correlated with obesity. We found that plasma FGF2 levels were positively correlated with fat mass, and marginally correlated with BMI in the same direction, suggesting that FGF2 might play a positive role in obesity. However, a previous study reported that serum FGF2 levels were negatively correlated with BMI, visceral fat and subcutaneous fat in a 30 Japanese population ${ }^{11}$. Such discrepancy might be due to the population specificity and the relatively small sample size of that study. Then, we hypothesized that association might also exist between the SNPs in FGF2 gene and obesity traits. Given the limited evidence of association study for human FGF2 gene with obesity, we investigated whether the common variants in FGF2 were associated with obesity phenotypes, including BMI, fat mass and WHRadjBMI. We identified one SNP (rs167428) significantly associated with fat mass both in our Chinese discovery and replication samples. Previous study by Shungin et al. reported a SNP (rs303084) near FGF2 as a new loci associated with WHRadjBMI ${ }^{12}$. This SNP was over $200 \mathrm{~kb}$ far away from FGF2. We examined LD between our identified SNP and the reported SNP using SNAP (data were derived from the CHBJPT panel of HapMap 3 (release 2 ) dataset) ${ }^{15}$. The pairwise LD was quite weak $\left(r^{2}=0.010, D^{\prime}=0.207\right)$, suggesting that our identified SNP could be a novel independent loci for obesity. To further assess the functional role of the identified SNPs, we performed eQTL analyses and found that SNPs associated with fat mass also affected mRNA expression level of FGF2 in human LCLs using public HapMap data. Taking into account of all those biological evidence and our statistical findings, we suggested that FGF2 gene could have potential impact on human fat mass regulation, and high plasma FGF2 level might lead to the increased risk of obesity.

Using the public available datasets on GWASs of obesity related traits, we sought for further evidence to support our findings. In the GIANT Consortium, although the SNP rs167428 was not validated, another SNP, rs308442, which was in modest LD with rs167428 $\left(r^{2}=0.31, D^{\prime}=1.0\right)$ and belonged to one block, was found to be associated with HIPadjBMI and WHRadjBMI in females. Moreover, a SNP (rs1048201) in 3'UTR of FGF2 was found to be associated with HIPadjBMI and WHRadjBMI, and also associated with T2D risk in the DIAGRAM Consortium. These two SNPs showed significant signals in our Chinese discovery sample. Since the subjects in the two large consortiums are all European ancestry individuals, the different LD patterns and population structures might cause the discrepancy of associated SNPs. However, all of these significant evidences, combined with our findings, support FGF2 as an obesity-associated gene in humans.

Our study samples were recruited from Midwestern area of China, where is relatively conservative and contains homogenous population. Using the same population, our previous published GWAS studies ${ }^{16,17}$ demonstrated that there was no population stratification in this population. Therefore, our association results are unlikely to be plagued by spurious associations due to population stratification.

Our study used quantitative obesity-phenotypes to test for genetic associations. When taking obesity as categorical outcome based on BMI (people who had the BMI $>27.5 \mathrm{~kg} / \mathrm{m}^{2}$ were considered obese according to the universal classification criterion of obesity of Asian), there were only 67 (5.2\%) and 100 (9.7\%) obese individuals in the discovery and replication cohorts, respectively. Since limited sample size with low statistical power might reduce the chance of detecting a true effect, analysis for association with obesity as categorical trait was not preferred in our study.

In our study, we did not find significant association with BMI. This may be due to the incomprehensive estimation of obesity by BMI. BMI is based on the observation that body weight is proportional to the squared height in adults with normal body frames ${ }^{18}$. This property makes BMI ignoring several important factors affecting adiposity. For instance, individuals with a normal BMI may suffer from sarcopenic obesity due to greater loss of muscle mass ${ }^{19}$. Fat mass derived from DXA, on the other side, is a direct measurement of fat, is more homogeneous and may reflect obese status more accurately. Therefore, fat mass can characterize obesity from different aspects and could be used as a complementary indicator for obesity.

In conclusion, our study provided novel evidence that human plasma FGF2 levels were positively correlated with fat mass, and genetic polymorphisms in FGF2 gene were associated with fat mass in Han Chinese population. Our results suggest that FGF2 could be a novel candidate for obesity by processing a series of association tests and referring to its biological functions. The evidence of the biological mechanism of FGF2 in body fat mass regulation is needed to confirm our findings in later studies. 


\section{Materials and Methods}

Subjects. The study was approved by the Institutional Review Board of Xi'an Jiaotong University and carried out in accordance with the approved guidelines. All the volunteers signed informed consent documents before entering the study.

There were three sample sets in our study. For the plasma FGF2 levels test, we included 62 unrelated subjects. For the SNP association test, we expanded our sample size to 1,300 subjects as the discovery phase, and an additional 1,035 subjects as the replication phase, which contained the 62 plasma sample subjects. All the subjects were unrelated northern Han Chinese adults that were recruited from the city of Xi'an and its neighboring areas. Subjects with chronic diseases and conditions that might potentially affect body mass, structure, or metabolism were excluded from the study to minimize the influence of known environmental and therapeutic factors on $\mathrm{BMI}$ and fat mass variation. These diseases/conditions included chronic disorders involving vital organs (heart, lung, liver, kidney, and brain), serious metabolic diseases (diabetes, hypo- and hyper-parathyroidism, hyperthyroidism, etc.), chronic use of drugs affecting body mass (hormone replacement therapy, corticosteroid therapy, anti-convulsant drugs), and malnutrition conditions (such as chronic diarrhea, chronic ulcerative colitis, etc.), etc.

Phenotype measurements. Waist and hip circumferences were measured with a calibrated tape measure. BMI values were calculated as body weight (in kilograms) divided by the square of height (in meters). Weight was measured in light indoor clothing without shoes, using a calibrated balance beam scale, and height was measured using a calibrated stadiometer. Body fat mass (in kilograms) was measured using dual energy X-ray absorptiometry (DXA) by Hologic $4500 \mathrm{~W}$ machines (Hologic Inc., Bedford, MA, USA) under strict protocols. The coefficient of variation $(\mathrm{CV})$ value of the DXA measurement for fat mass was approximately $1.1 \%$. In this study, we included BMI, body fat mass and WHRadjBMI, to assess obese status ${ }^{12,20}$.

Plasma FGF2 concentrations measurement. Fasting blood samples were obtained in the morning from 8:00 to 10:00 AM. Plasma samples were collected by blood separation with centrifugation at 2,500 rpm for $10 \mathrm{~min}$ at $4{ }^{\circ} \mathrm{C}$, following both the manufacturer's protocol and specialized laboratory assay quality control procedures, and the samples were saved at $-80^{\circ} \mathrm{C}$ until analysis (no longer than 6 months). FGF2 concentrations were measured through enzyme-linked immunosorbent assay (ELISA) using ELISA commercial kit (RapidBio, West Hills, CA, USA) strictly under the manufacturer's protocol. According to the instruction, the plate coefficient of variation was less than $15 \%$, the assay range was 1.0 to $100.0 \mathrm{ng} / \mathrm{L}$, and the sensitivity was less than $1.0 \mathrm{ng} / \mathrm{L}$. No soluble structural analogues with other cross-reaction were observed during the whole procedure.

SNP selection and genotyping. Genomic DNA was extracted from peripheral blood leukocytes using a commercial isolation kit (Gentra systems, Minneapolis, MN, USA) following the protocol of the kit. We explored the dbSNP (http://www.ncbi.nlm.nih.gov/SNP) and HapMap (http://hapmap.ncbi.nlm.nih.gov/cgi-perl/gbrowse/ hapmap28_B36/) databases searching SNPs in FGF2. We selected 11 SNPs spanned the genic region in FGF2 according to the following criteria, in order of importance in our selection scheme: (i) minor allele frequency $(\mathrm{MAF})>0.05$ in the CHB population; (ii) an average density of 1 SNP per $7 \mathrm{~kb}$; (iii) tagging SNPs are preferential; (iv) potential functional SNPs.

For the discovery sample, SNPs genotyping was carried out using MALDI-TOF mass spectrometry on a MassARRAY system (Sequenom, Inc., San Diego, CA) with iPLEX assay. Genotype calling was performed in real time with MassARRAY RT software version 3.0.0.4 and analyzed using the MassARRAY Typer software version 3.4 (Sequenom). Genotyping quality control procedures leading to SNP exclusion were call rate $<90 \%$, $\mathrm{MAF}<0.05$ and $P<0.001$ for deviations from Hardy-Weinberg equilibrium (HWE). The average call rate was $98 \%$ and the duplicate concordance rate was $99 \%$. All of the 11 SNPs in the discovery sample were successfully genotyped and used for association analyses. Then, we selected the top 4 significant SNPs in the discovery phase to perform replication study. SNPs genotyping used in the replication sample was the same as that adopted in the discovery sample. Of the 4 SNPs attempted, one SNP (rs1449683) failed in the replication genotyping procedure.

Statistical analyses. The raw BMI and fat mass values were adjusted with covariates including sex and age. The residuals were used for further association analyses. For the plasma FGF2 analyses, we used linear regression model to assess the relationship of plasma FGF2 concentrations with obese status and genetic variants separately. These procedures were conducted with Minitab 14 software (PA, USA). For the SNP association analyses, linear regression implemented in PLINK ${ }^{21}$ was fitted to test for associations under the additive inheritance model in the discovery sample and the replication sample respectively. Population haplotypes and their frequencies were inferred using Haploview software ${ }^{22}$. Heat plot of linkage disequilibrium using $r^{2}$ value was also generated by Haploview $^{22}$. Haplotypes with estimated frequencies greater than $5 \%$ were included for association analyses by PLINK $^{21}$. A raw $P$ value of $<0.05$ in our study was considered nominally significant, and the Bonferroni correction was used for multiple testing corrections. Finally, the significance threshold was set as $P<0.0045$ in the discovery sample for single SNP test $(0.05 / 11$ SNPs that were included in the association analyses) and $P<0.0056$ for haplotype analysis (0.05/9 haplotypes that were included in the association analyses).

Meta-analysis statistics and heterogeneity test were generated using METAL software package (http://genome. sph.umich.edu/wiki/METAL_Documentation). The meta-analyses were performed with the inverse of the corresponding standard errors weighted effect size, and the Cochran's Q-test was implemented for heterogeneity.

Expression quantitative trait locus (eQTL) analysis. To explore whether the identified SNPs correlated with the transcription variants, we conducted association analysis between SNPs and mRNA expression levels of the FGF2 gene. Gene expression data were derived from human lymphoblastoid cell lines (LCLs) of 210 unrelated individuals from HapMap populations in the NCBI Gene Expression Omnibus ${ }^{23,24}$. The sample comprised $60 \mathrm{CEU}, 45 \mathrm{CHB}, 45$ JPT and 60 YRI. SNP genotype data were derived from the corresponding HapMap Phase 
III dataset. The associations between expression levels and SNPs were examined using PLINK ${ }^{21}$ through linear regression model.

\section{References}

1. Kopelman, P. G. Obesity as a medical problem. Nature 404, 635-43 (2000).

2. Consultation, W. H. O. E. Appropriate body-mass index for Asian populations and its implications for policy and intervention strategies. Lancet 363, 157-63 (2004).

3. Stunkard, A. J., Foch, T. T. \& Hrubec, Z. A twin study of human obesity. JAMA 256, 51-4 (1986).

4. Maes, H. H., Neale, M. C. \& Eaves, L. J. Genetic and environmental factors in relative body weight and human adiposity. Behav Genet 27, 325-51 (1997).

5. Loos, R. J. \& Bouchard, C. FTO: the first gene contributing to common forms of human obesity. Obes Rev 9, 246-50 (2008).

6. Zhao, J. et al. The role of obesity-associated loci identified in genome-wide association studies in the determination of pediatric BMI. Obesity (Silver Spring) 17, 2254-7 (2009).

7. Crandall, D. L., Hausman, G. J. \& Kral, J. G. A review of the microcirculation of adipose tissue: anatomic, metabolic, and angiogenic perspectives. Microcirculation 4, 211-32 (1997).

8. Mydlo, J. H., Kral, J. G. \& Macchia, R. J. Preliminary results comparing the recovery of basic fibroblast growth factor (FGF-2) in adipose tissue and benign and malignant renal tissue. J Urology 159, 2159-2163 (1998).

9. Kawaguchi, N. et al. De novo adipogenesis in mice at the site of injection of basement membrane and basic fibroblast growth factor. Proc Natl Acad Sci USA 95, 1062-1066 (1998).

10. Kakudo, N., Shimotsuma, A. \& Kusumoto, K. Fibroblast growth factor-2 stimulates adipogenic differentiation of human adiposederived stem cells. Biochem Biophys Res Commun 359, 239-44 (2007).

11. Seida, A. et al. Serum bFGF levels are reduced in Japanese overweight men and restored by a 6-month exercise education. Int $J$ Obes Relat Metab Disord 27, 1325-31 (2003).

12. Shungin, D. et al. New genetic loci link adipose and insulin biology to body fat distribution. Nature 518, 187-96 (2015).

13. Morris, A. P. et al. Large-scale association analysis provides insights into the genetic architecture and pathophysiology of type 2 diabetes. Nat Genet 44, 981-90 (2012).

14. Hutley, L. J. et al. A putative role for endogenous FGF-2 in FGF-1 mediated differentiation of human preadipocytes. Mol Cell Endocrinol 339, 165-71 (2011).

15. Johnson, A. D. et al. SNAP: a web-based tool for identification and annotation of proxy SNPs using HapMap. Bioinformatics 24, 2938-9 (2008).

16. Guo, Y. et al. Genome-wide association study identifies ALDH7A1 as a novel susceptibility gene for osteoporosis. PLoS Genet 6, e1000806 (2010)

17. Yang, T. L. et al. Genome-wide copy-number-variation study identified a susceptibility gene, UGT2B17, for osteoporosis. Am J Hum Genet 83, 663-74 (2008).

18. Quetelet, L. A. A treatise on man and the development of his faculties. 1842. Obes Res 2, 72-85 (1994).

19. Shah, N. R. \& Braverman, E. R. Measuring adiposity in patients: the utility of body mass index (BMI), percent body fat, and leptin. PLoS One 7, e33308 (2012).

20. Heid, I. M. et al. Meta-analysis identifies 13 new loci associated with waist-hip ratio and reveals sexual dimorphism in the genetic basis of fat distribution. Nat Genet 42, 949-60 (2010).

21. Purcell, S. et al. PLINK: a tool set for whole-genome association and population-based linkage analyses. Am J Hum Genet 81, 559-75 (2007).

22. Barrett, J. C., Fry, B., Maller, J. \& Daly, M. J. Haploview: analysis and visualization of LD and haplotype maps. Bioinformatics 21, 263-5 (2005).

23. Stranger, B. E. et al. Relative impact of nucleotide and copy number variation on gene expression phenotypes. Science 315, 848-53 (2007).

24. Stranger, B. E. et al. Population genomics of human gene expression. Nat Genet 39, 1217-24 (2007).

\section{Acknowledgements}

This work was supported by the National Natural Science Foundation of China (31371278, 31471188, 81573241, 31301029, 31511140285), China Postdoctoral Science Foundation (2015M570820), Natural Science Basic Research Program Shaanxi Province (2015JQ3089), and the Fundamental Research Funds for the Central Universities.

\section{Author Contributions}

T.L.Y. and Y.G. conceived and supervised the project. R.H.H., D.L.Z., X.F.C. and J.B.C. performed the experiments. R.H.H. and S.S.D. conducted data analysis. G.Z. W. and H.Y. contributed to experiments. All authors discussed the results and commented on the manuscript.

\section{Additional Information}

Competing financial interests: The authors declare no competing financial interests.

How to cite this article: Hao, R.-H. et al. Associations of Plasma FGF2 Levels and Polymorphisms in the FGF2 Gene with Obesity Phenotypes in Han Chinese Population. Sci. Rep. 6, 19868; doi: 10.1038/srep19868 (2016).

(c) (i) This work is licensed under a Creative Commons Attribution 4.0 International License. The images or other third party material in this article are included in the article's Creative Commons license, unless indicated otherwise in the credit line; if the material is not included under the Creative Commons license, users will need to obtain permission from the license holder to reproduce the material. To view a copy of this license, visit http://creativecommons.org/licenses/by/4.0/ 